\title{
Improvements for 802.11-based Location Fingerprinting Systems
}

\author{
Hendrik Lemelson, Stephan Kopf, Thomas King, and Wolfgang Effelsberg \\ Department of Computer Science \\ University of Mannheim, Germany \\ \{lemelson,kopf,king,effelsberg\}@informatik.uni-mannheim.de
}

Position estimation with 802.11 and location fingerprinting has been a topic in research for quite some time already. But there are still some unaddressed issues that are the reason why such systems are not widely used. First, the positioning accuracy still leaves space for improvements. Second, users generally have no information about the quality of the estimated position. Especially in cases where the positioning error is large, the user's trust in the system suffers if he is not notified. Third, most systems that rely on a location fingerprinting approach need a time and effort consuming setup phase in which the training data has to be collected and processed. In this paper, we give an overview of existing possibilities to improve location fingerprinting systems under each of these three aspects. Several alternative solutions for each problem are presented. Finally, a discussion gives an overall picture of the usability of the solutions when considering the system as a whole.

\section{INTRODUCTION}

Nowadays, as the development of mobile devices continues, numerous applications and services for nomadic users become possible that might have been utopian a decade ago. With high processing power, long-lasting batteries and high bandwith wireless data connections, people can browse the Web at any time, send and receive email, watch high-resolution video streams, or even broadcast information and media-content from their own device.

But whereas all these thrilling applications are known from stationary computers at home or at work, there exists also another type of services that is exclusive to mobile users: the so-called location-based services (LBSs). LBSs are a special category of the context-aware services because they do not consider the complete available context information of a user to improve their delivered service but are confined to only using information about the user's current whereabouts. While this dramatically reduces the service complexity, it generally leverages the usefulness of the offered service a lot.

When a user is located outdoors, the most prominent source for location information used by LBSs are satellite-based systems like the Global Positioning System (GPS) [1]. But whereas these systems offer a good position accuracy under optimal conditions - like in a suburban open-sky environment - they fail to deliver precise position estimates in cases where the mobile device has no line of sight to at least three satellites. Unfortunately, this is the case in those places where users spend most of their time: indoors and within metropolitan areas where large buildings shade the mobile device from the signals of the satellites.

Several approaches exist that try to overcome these limitations. For instance, to estimate a user's position the RightSPOT [2] positioning systems uses the FM signals of radio stations, Place Lab [3] uses the IDs of radio beacons like GSM cells or 802.11 access points (APs), and the system developed by Ubisense $^{1}$ performs time-difference of arrival (TDOA) measurements of wideband pulses sent by proprietary base stations. But whereas all of these systems offer an alternative to GPS at first sight, they all suffer from coarse position accuracy or the requirement for special hardware.

For the position estimation in such adverse situations where satellite-based systems are not available, location fingerprinting (LF) has emerged as a very promising alternative. With LF, depending on the system setup, e.g., the signals of 802.11 access points (APs) or GSM cells can be used to reliably estimate a user's position. To achieve this, LF systems use a two-stage approach. In the first stage - often called training phase - the system operator creates fingerprints at selected reference spots within the area the system shall cover and stores them in a database together with the coordinates or an identifier for the location where they were collected. Each fingerprint reflects the unique properties of the signal space at its specific position and can incorporate e.g., the set of receivable APs, the average signal strength of each AP or a signal strength distribution for each AP. During the second stage - the position determination phase - the mobile device creates the equivalent of a fingerprint at the yet unknown position of the user. The positioning system then compares this fingerprint to all the fingerprints stored in the database and selects - according to a system-specific metric - the bestmatching counterpart. Basically, the coordinates or the location of that counterpart are then returned to the user as a position estimate.

A good overview and taxonomy of 802.11-based LF systems

\footnotetext{
${ }^{1}$ http://www.ubisense.net
} 


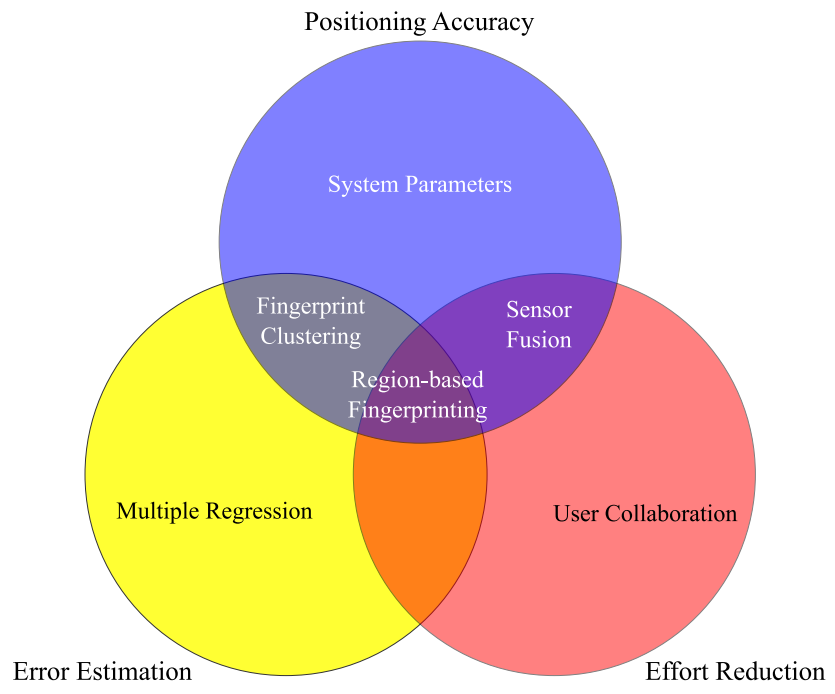

Fig. 1: Overview of the issues and suggested solutions for 802.11-based LF systems addressed in this paper.

as well as also distinctive feature of LF systems can be found in [4].

Regarding the metric used for finding the best-matching fingerprint in the database, two major classes of algorithms exist: deterministic ones and probabilistic ones. While especially systems that use probabilistic algorithms offer a competitive position accuracy in most cases, they still have some drawbacks. The first is the uncertainty that is still inherent in the position estimation. Even besides their good average position accuracy, there exist cases where the conditions are suboptimal and the accuracy rapidly decreases. As most LF systems do not offer means to estimate the error that has to be expected, in these cases the user is not notified that the currently estimated position might be far from his real whereabouts.

The second drawback is the need for the very time consuming creation of the fingerprint database. To achieve a satisfactory positioning accuracy, a small-spaced grid of reference spots has to be laid out over the area of operations, and at each of these spots a high number of training measurements have to be made [5]. This is one of the reasons why LFbased positioning systems have not yet been widely adopted for general use.

The goal of this paper is to give the reader an overview of existing possibilities to improve the - from a system operator side - most important aspect of LF systems, namely the effort needed for setup. Additionally, the paper also points out ways to improve the positioning accuracy of LF system and to estimate the position error that has to be expected. These are important issues especially from a user perspective (see Figure 1).

Even though the concepts presented are mostly universal, we will mainly stick to systems that use 802.11 as the underlying radio infrastructure. The reason here is that nowadays 802.11 still is the most ubiquitous radio technology that offers the possibility of a position accuracy sufficient for most indoor scenarios, a sufficient coverage, and is easily usable.

The remainder of the paper is structured as follows: The following section gives a short overview on LF systems from past and current research. Section III then introduces current methods from other researchers as well as from ourselves to improve the position accuracy. This is followed by Section IV where we present techniques to estimate the error of common LF systems. Subsequently, Section V gives an overview on approaches to reduce the effort for creating the fingerprint database. In Section VI, we discuss the presented solutions from a more general perspective before we, in Section VII, finally conclude the paper and give some directions for future work.

\section{RELATED WORK}

The development of positioning systems still is a very active topic in the research community. During recent years, many systems have been proposed to estimate a user's position. These systems use different techniques like infrared light [6], ultra-sonic pulses [7], or radio signals [8] to fulfill the task of position determination. All of these systems are mainly targeted at indoor usage and even if some alternative approaches exist to estimate a user's position outdoors, there is much less activity in this sector due to the predominance of the satellitebased GPS [1].

The advantage of systems that use radio signals is that these signals are not stopped by walls and, especially when using 802.11, that the infrastructure is already available in most places where people live and work (see [3] and [9]).

One of the problems these systems have to face is the propagation behavior that radio signals have inside buildings. Generally, we have to cope with diffraction, scattering, shading and multipath propagation which make it very hard to create proper propagation models and to anticipate the signal's properties at a certain position in space and over time [10]. Therefore, many novel systems nowadays use the location fingerprinting approach.

Considering the different classes of metrics used for LF systems, a representative from the class of systems that use deterministic metrics is the RADAR [11] positioning system that also was the first to incorporate the concept of LF. RADAR uses the Euclidean distance in signal space to determine the similarity of two fingerprints.

The class of systems that use a probabilistic metric generally offers a better positioning accuracy in most cases [12] The system introduced by Haeberlen et al. [13] is a typical representative for this class. Given the current measurements, this system computes a probability for each fingerprint of the user being located at the position the fingerprint represents. This is done using a hidden Markov model and matching the signal strength values collected at the user's current position to signal strength distributions stored in the fingerprints from the database. The fingerprint that offers the highest probability is selected, and the position belonging to that fingerprint is returned to the user as a position estimate. 
Inspired by the fundamental research on this topic, other systems have been proposed that extend the basic LF approach by e.g., measurement pre- or postprocessing steps to increase the accuracy or to reduce the training effort. An example for such an advanced system is Horus [14] that uses an algorithm to detect and eliminate small-scale signal variations like they often occur when measuring the signal strength of an 802.11 access point at a fixed position.

\section{Positioning ACCURACY}

Besides the mere variation of the metric, several other approaches exist to improve the positioning accuracy of LF systems based on 802.11. The possibilities range from advanced pre- or post-processing steps of the sensor data over the consideration of the position or measurement history to the use of sensor fusion techniques.

\section{A. Basic System Parameters}

The easiest way to improve the positioning accuracy of LF systems is to modify the system's setup parameters. As shown in [5], a reduction of the grid spacing between the reference spots - in case a grid is used - has a major influence on the achievable accuracy. In addition, the number of measurements used to create each single fingerprint during the training phase as well as the number used during the position determination phase for the position estimation have a large impact on the positioning accuracy. As a consequence, choosing unsuitable parameters decreases the accuracy of the positioning system significantly.

\section{B. Measurement Processing}

To improve the position accuracy of 802.11-based LF systems further, an often suggested possibility is the use of advanced machine learning algorithms. In [15], for instance, a particle filter approach is chosen to estimate the position of a user based on signal strength measurements. Particle filters are part of the sequential Monte-Carlo methods and quite similar to Kalman filters. They are very well suited to deal with data and measurements that do not adhere to standardized distributions like e.g., Gaussian distributions, as is the case for indoor signal strength measurements. Also, particle filters are computationally less expensive than many other methods. This makes them especially suitable for the use on mobile devices, being less powerful compared to stationary computers.

Widyawan et al. use a variation of the general particle filter approach called backtracking particle filter to increase the positioning accuracy of the underlying LF system.

\section{Feature Selection}

Another approach is used by SkyLoc [16]. SkyLoc is a localization system designed to run on a mobile phone and used to reliably determine the floor on which a user is located in tall multi-story buildings. It uses the radio signals from GSM cells and location fingerprints created for each floor of the building. To find the best-matching fingerprint in the database, SkyLoc relies on a deterministic approach - namely the Euclidean distance in signal space.

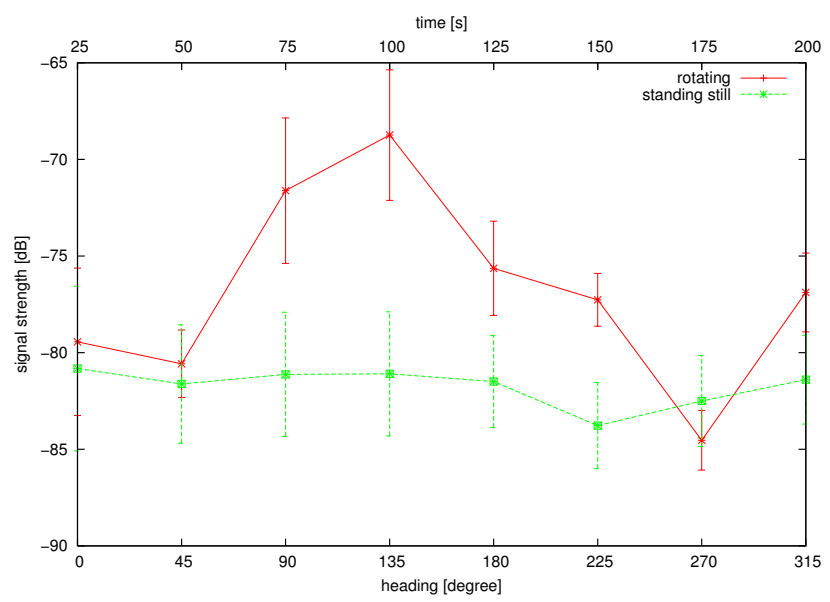

Fig. 2: Influence of the user's body on the received signal strength [17].

To additionally improve the estimation accuracy, SkyLoc incorporates a method called feature selection. Instead of using data for all GSM cells that were received at the user's current position, only a subset of these is used for matching against the fingerprints in the database. To identify the subset that offers the highest position accuracy in advance of the deployment, the authors of [16] suggest three different methods: The first one is called Forward Selection. Starting with an empty set, the feature that offers the highest increase in average position accuracy is added to the set of used features until it has reached its final size. The second alternative is Backward Elimination. It proceeds the opposite way. From the full set of all available features, successively those are removed from the set of which their removal offers the highest increase in the average position accuracy. Finally, the third method, namely Per-Floor Feature Selection, tries to find not one optimal feature set to be used for the whole database but optimizes the set for each story separately. Additionally, this third method also uses weights for the single features to further increase the performance.

\section{Sensor Fusion}

A further improvement of the positioning accuracy can be achieved by sensor fusion. Sensor fusion means to not only use the readings from one single data source, in our case for instance an 802.11 network card, but also to consider data from other sources. This can be cameras, other radio devices like Bluetooth peripherals, audio sensors or even systems used for the shock protection of computer harddrives. An example for a system that follows this approach is Compass [17]. Compass is based on LF with 802.11 and exploits the fact that the human body strongly attenuates 802.11 radio waves that pass through it (see Figure 2) which can highly deteriorate the positioning accuracy if the user is standing in an unsuitable position. The Compass system creates not only one fingerprint for each reference spot but a set of fingerprints with the user heading in different directions. The directions are predefined, and the adjustment is done using a digital compass built into the mobile device. In the position determination phase, the 


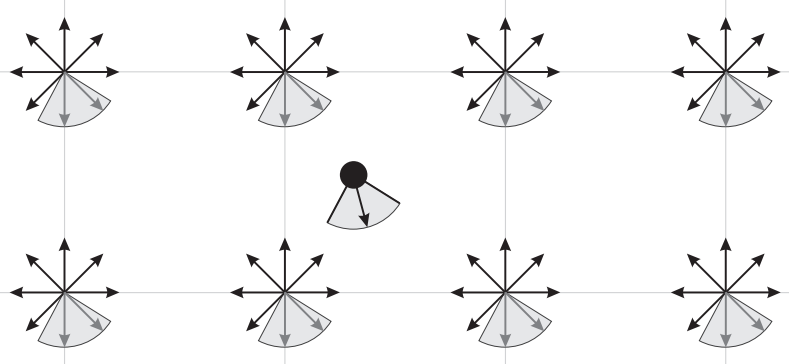

Fig. 3: Selection of the subset of used fingerprints based on the user's heading [17]. The arrows represent fingerprints taken while facing in the direction of the arrow. The black dot with the arrow shows the user and the direction he is currently facing. Finally, the grey area represents the intervall used to select appropriate fingerprints for the position estimation.

user's mobile device - as well equipped with a digital compass - creates a fingerprint of the current radio environment as usual. This fingerprint is not matched against all fingerprints in the database, but only against those that have a similar orientation as the user (see Figure 3). Using this sensor fusion approach, a significant accuracy improvement of $\approx 20 \%$ is achieved.

\section{E. Sampling Frequency}

While it does not completely fit into the category of improving the accuracy, at this point also Composcan should be mentioned. Composcan is a system that, based on the variance of subsequent measurements taken during the position determination phase, can estimate whether the user is moving or standing still. The reasons why such an approach can also be used to increase the positioning accuracy are twofold. First, when the user is detected as being stationary, several consecutive measurements taken at the same position can be combined before comparing them to the fingerprints in the database. As shown in [5], increasing the number of measurements used for the position estimation remarkably increases the positioning accuracy. Second, it is well known that the sampling of measurements with 802.11 network cards interferes with ongoing data communication. Therefore, the measuring frequency as well as the measurement scheme can be adapted for a stationary user to reduce the impact on concurrent data communication. If the user is moving the sampling frequency is raised again. This helps to maintain an acceptable position accuracy at the cost of decreased data throughput.

\section{ERROR ESTIMATION}

Even though reducing the overall error of LF systems is a very desireable goal, the achievable accuracy will probably always leave room for improvements. When considering the average accuracy of current systems which is typically between 2.5 meters and 3 meters, small positioning errors are acceptable for most applications. One of the remaining major problems with the position estimation is the so-called long-tail

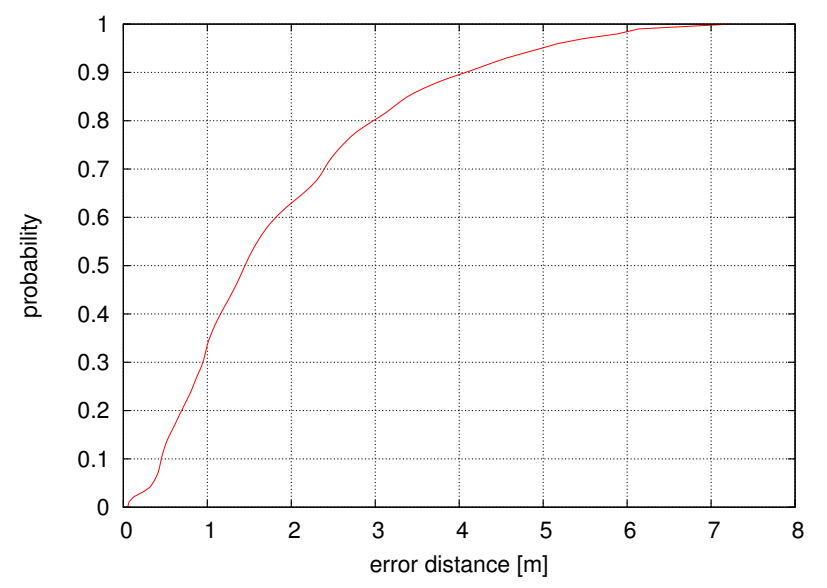

Fig. 4: Example for a cumulative error distribution of an LF system. Here, for instance, the error is smaller than $3 \mathrm{~m}$ in $80 \%$ of all cases.

error: the quite unlike but still existing occurrence of a very large error. Especially for cases in which these errors occur, an estimation of the positioning error would be of high value to the user. In this section, we therefore introduce different approaches that estimate the error of LF systems.

\section{A. Error Distribution}

The most basic approach of error estimation is to supply the user with general information about the position error that has to be expected when using a certain positioning system. This can be achieved by, for instance, supplying him with a cumulative error distribution of the positioning system (see Figure 4). The user then can estimate the probability of a certain error to happen and adapt his behavior accordingly.

Even this very simple way of dealing with error information already has a remarkable influence on the user perception of an LBS. As a study by Dearman et al. [18] has shown, even this unspecific knowledge of the error distribution helps users of an LBS to perform better when e.g., solving location-specific tasks.

The main drawback of this approach is that it only offers a general and not a situation-specific error estimation.

\section{B. Multiple Regression}

The authors of [18] also introduced the use of multiple regression to estimate the position error. Using the training data and a smaller amount of test measurements for the reference spots, the system operator computes a linear model of the error that has to be expected based on a defined set of signal features. This set can contain the receivable access points or GSM cells, the signal strengths, properties of the noise floor or other parts of the measurements. During the position determination phase, the user then can apply the linear model to his currently collected data and by such compute an error estimate.

While this approach already offers promising results, it uses quite basic features to estimate the error. The next two methods 


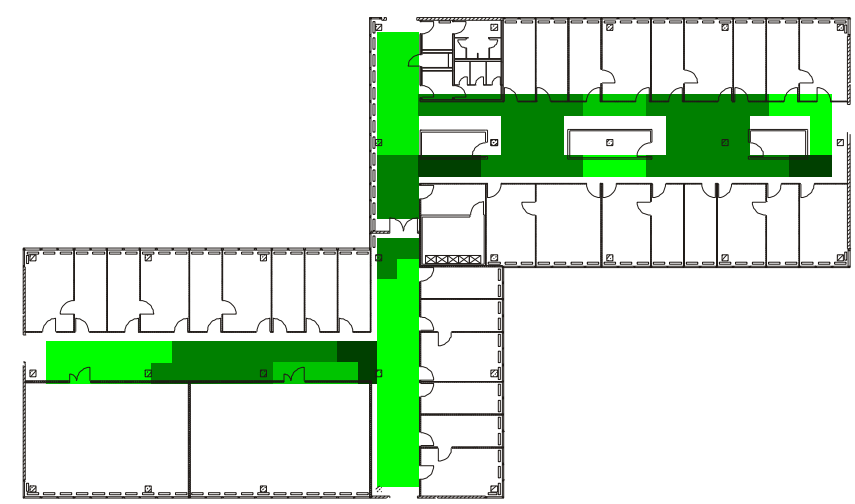

Fig. 5: Example for a region map computed by the fingerprint clustering algorithm.

take a similar direction but use more advanced features to accomplish their task.

\section{Fingerprint Clustering}

Introduced by ourselves in [19], the fingerprint clustering approach is an algorithm that estimates the position error based only on the training data. To estimate the error that has to be expected at a certain position, the algorithm uses the training data to identify consecutive areas of similar signal properties. This is done by first laying out a grid of cells over the area to cover. Each cell, in the beginning, is a single-cell cluster. Then, physically adjacent clusters that are similar according to a similarity measure are merged together to larger clusters. This is continued until, due to the similarity measure, no further merges are possible. The result is a set of physically connected clusters of which each represents a region of similar signal properties (see Figure 5). The information about this final set of clusters is stored for later reference. After a position estimate has been computed during the position determination phase, the algorithm checks in which of the identified regions the estimated position lies and uses the dimensions of that region to compute the position error that is to be expected.

\section{Best Candidate Set}

Also introduced in [19], the best candidate set algorithm also uses an advanced set of features and knowledge about the internal procedures of LF algorithms to make reliable error estimates. Taking an 802.11-based LF algorithm that uses a probabilistic metric as a baseline, the best candidate set algorithm exploits the fact that the algorithm during the position determination phase has knowledge not only of the most probable reference spot but also of the probabilities for all other spots. The best candidate set algorithm considers this by using the following approach. First, the set of the $n$ most probable fingerprints is formed. Then, the distance between the position of most probable fingerprint and the other $n-1$ fingerprints is computed. Finally, the average of these distances is returned as an error estimate. Besides using the average distance, also other options like using the maximum distance were considered for the best candidate set algorithm but these did not lead to better results. The value suggested for $n$ is 3 .

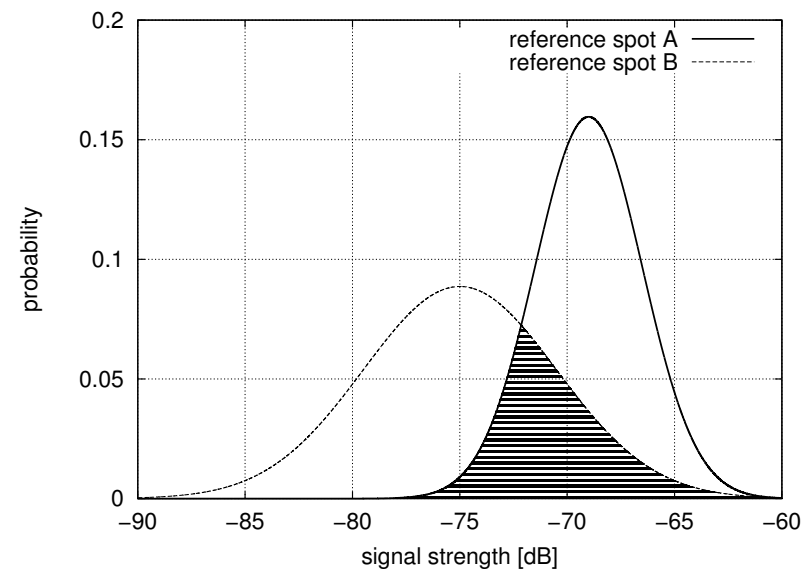

Fig. 6: Overlapping signal strength distributions for one access point at two adjacent reference spots.

\section{EFFORT REDUCTION}

It becomes clear when regarding the requirements of most LBSs that - though undoubtfully desireable - an improvement of the accuracy as well as the very precise estimation of the position error is only necessary in a few cases. The reason why LF still is only rarely adopted for position estimation beyond academia is the need for the very time-consuming setup of the system. As we have analyzed in [20], a finegrained grid of reference spots has to be used, and at least 20 measurements have to be collected at each reference spot to achieve an accuracy sufficient for most LBSs.

\section{A. Quick Fingerprinting}

Quick fingerprinting [21] tries to reduce the calibration effort by reusing collected training measurements. Especially for smaller distances between the reference spots in the grid, the inevitable variation in the 802.11 radio signals generally results in an overlapping of the received signal strength distributions for one access point at adjacent reference spots (see Figure 6).

Quick fingerprinting exploits this by not only using samples that were collected at the current reference spot, but also the samples from the adjacent reference spots to create the fingerprint. Doing so, the number of measurements that have to be collected at each single spot can be reduced by $\approx 80 \%$ without losing positioning accuracy.

\section{B. Region-based Fingerprinting}

Region-based fingerprinting goes even one step further than quick fingerprinting. Measurements collected at different positions are combined into one fingerprint, too. But instead of using samples from adjacent reference spots, the samples in this case are collected while walking through the area of operations on pre-defined trajectories. The algorithm then in a similar manner as described in Section IV-C - combines similar measurements to consecutive regions of similar signal properties. Thus, the amount of training measurements can be reduced, and the way the training measurements are collected 
becomes much easier. This has to be traded for a minor loss in positioning accuracy.

\section{User Collaboration}

The probably most effort-saving way to setup a LF system is to let the user provide the training data for the fingerprint database on the fly. While with this approach the system operator loses control over the training data collection, he gains the possibility to continuously improve the coverage and to keep the database updated.

A good example for an LF systems that follows this approach is Redpin [22]. It uses a software that runs on the mobile device to collect signal measurements from GSM cells, 802.11 APs and nearby Bluetooth devices. Besides the normal operation where a fingerprint created from live measurements is matched against the fingerprints in the database, the software offers the possibility to store a fingerprint for the current location in the database in case no matching fingerprint was found and therefore no position estimation was possible. Thus, different users can collaborate with the system operator to extend the system to new areas and to keep the available data up-to-date. This reduces the training effort of the system operator to a minimum.

Another example for a system that uses user-supplied data was introduced by Chai and Yang in 2005 [23]. In contrast to Redpin [22], it does not completely rely on the user-supplied data but requires an initial fingerprint database set up by the system operator. During the position determination phase, socalled unlabeled traces - sequences of user measurements of which the positions are unknown - are used to refine the data in the fingerprint database. To identify the corresponding fingerprints, the authors apply an algorithm introduced by Dempster et al. [24] called EM-algorithm. This algorithm iteratively computes maximum likelihood estimates from a given set of incomplete observations and is split in two steps, an expectation step and a maximization step. The advantage of using unlabeled traces compared to Redpin is that the operator can offer a guaranteed coverage of the area the basic fingerprint database covers. But this also shows the limits of this approach because the unlabeled traces can only be used to refine existing data, and the users cannot extend the coverage area of the system on their own.

\section{Generated Fingerprints}

It is also possible to computationally create the fingerprint database. This approach can very effectively reduce the effort to set up an LF system. Most common here is the use of a radio propagation model in combination with a few or even only one reference measurement. Based on these measurements and the model, a radio map for the area to cover is computed. With values taken from this computed radio map, fingerprints are created for each reference spot in the area to cover and stored in the fingerprinting database. The system introduced by Ji et al. [25] follows this concept.

Whereas such an approach very effectively reduces the effort, it is questionable how usable the system really is in practice. Considering the very chaotic behavior that radio signals show inside buildings, the performance of a system strongly depends on the quality of the radio model: If the used model does not reflect the real radio propagation correctly, the entire system will be unusable.

\section{E. Fingerprint Distribution}

Up to now, we have only presented approaches to reduce the effort for setting up an LF system. Another type of effort reduction comes very handy especially when dealing with LF systems on resource-constrained mobile devices.

One problem that large deployments of LF systems have to deal with is the size of the fingerprint database. As is often the case because of privacy considerations, the estimation of the user's position shall be calculated on his mobile device so that his position is not known to the service provider. To achieve this, the database containing the fingerprints has to reside on the mobile device, too. Especially for large deployments, this can present a problem for the restricted resources of a mobile device. Therefore, in [26] algorithms are presented to supply only the currently needed fraction of the fingerprint database to the user's mobile device. By chosing an adequately sized fraction, the user's privacy requirements can be fulfilled and the memory consumption can be reduced.

To split the database into meaningful parts, two algorithms that serve different memory requirements are presented in [26]. The first one is called Union of Access Points. Here the device requests all fingerprints in which any of the currently received APs is contained. This variant requires most memory on the mobile device. The second introduced algorithm named Intersection of Access Points only transmits those fingerprints to the mobile device that contain all APs received at the user's current position. This variant uses the least amount of memory.

\section{DISCUSSION}

We have shown in the previous sections that several different solutions exist to improve the drawbacks of 802.11-based LF systems. Each of these solutions fulfills its purpose when considering only its specific area. But a look at the complete picture shows that not all solutions are equally well suited for a real system.

\section{A. Positioning Accuracy}

For example, if we consider the Compass system presented in Section III-D, we can see that it offers a very competitive accuracy. But because one fingerprint has to be created for each direction considered, the effort to setup the system would be even larger than it already is for a basic LF system.

Exactly the opposite is true for the system presented in Section III-E. As this system only relies on data that is available anyway and as its usage would have no influence on other system parameters like the effort needed for setup, it can well be adopted for widespread usage in LF system. 


\section{B. Error Estimation}

As mentioned already in Section IV, the estimation of the expected error is mainly desireable to inform the user in case of possible large errors. This helps to increase the user's trust in the system. Because this is only possible with situationspecific knowledge, using only a distribution function like the one presented in Section IV-A seems inappropriate.

Better suited is the use of the Best Candidate Set algorithm presented in Section IV-D. The algorithm implicitly uses both information about the training and current data to create a reliable error estimation.

\section{Effort Reduction}

Finally, also the algorithms presented in Section $\mathrm{V}$ have both advantages and disadvantages. Whereas the system presented in Section V-D very consequently reduces the effort necessary for the setup of the system, this might come at the cost of a heavily degraded accuracy if the selected radio model is not suitable.

The system described in Section V-C seems to be much better suited. The effort is very effectively reduced while the accuracy of the system is only influenced in terms of missing coverage.

\section{Application Requirements}

As stated, not all solutions are equally well suited to solve their corresponding problem. Additionally, also the combination of different approaches is not always easily possible. Therefore, for a real deployment, the application requirements have to be considered as well to identify features that need special attention.

If we, for instance, think of a friend finder application that is used on a university's campus, on the one hand the accuracy could very well be reduced to room level as friends would be easily recognizable inside of a single room. The same is also be true for the error estimation, where only errors beyond the size of a room would matter to the user. On the other hand, as the campus of a university generally covers a large area, effort reduction here is a very important factor.

In contrast, when considering a warehouse navigation system, the situation is totally different. To locate possibly small items within the storage shelves, a very high accuracy is necessary. The estimation of the position error might also gain high importance in such a case as the system could also be used to verify access requirements. An example is an inference algorithm that, based on the position history, checks that an employee does not stay within a hazardous environment for too long. Such an algorithm obviously should only consider position estimates with a very low estimated error to avoid false alerts. Compared to the first two properties, the effort necessary for setup and operation is only of secondary importance in such a limited scenario.

\section{CONCLUSION}

In this paper we gave a brief overview of techniques used to address the major issues that are still obstacles to the wide adoption of location fingerprinting.
Firstly, we have presented different techniques that can be used to increase the positioning accuracy of 802.11-based LF systems. Increasing the accuracy is a prerequisite to be able to also allow the use of advanced LBSs. Secondly, several algorithms from the field of error estimation for location fingerprinting systems have been introduced. While this field of research is still quite new, it nevertheless is of high importance - especially to the users of LBSs. Subsequently, we have presented techniques that system operators can use to reduce the effort that is necessary to setup a location fingerprinting database. This can help system operators to deploy and maintain LF systems at reasonable costs and thus to make their usage feasible. During our discussion, we have finally shown, that whereas suitable solutions exist for each single problem, a combination of these is neither always possible nor always necessary. Which of the different parameters should be optimized is highly dependent on the targeted application.

In the future, several directions for further research are open. Besides the ongoing optimization of algorithms for the single problems - possibly with a focus on error estimation and effort reduction - also the development of algorithms that offer an adjustable solution to all issues might be considered. Additionally, also more effort is necessary to further understand the requirements of applications and scenarios in which LF with 802.11 is used to be able to even better tailor upcoming systems to the requirements of real users.

\section{REFERENCES}

[1] E. Kaplan and C. Hegarty, Understanding GPS: Principles and Applications, 2nd ed. Artech House Incorporated, Dec. 2005.

[2] J. Krumm, G. Cermak, and E. Horvitz, "RightSPOT: A Novel Sense of Location for a Smart Personal Object," in Proc. of the 5th Int. Conf. on Ubiquitous Computing (Ubicomp), Oct. 2003.

[3] A. LaMarca, Y. Chawathe, S. Consolvo, J. Hightower, I. Smith, J. Scott, T. Sohn, J. Howard, J. Hughes, F. Potter, J. Tabert, P. Powledge, G. Borriello, and B. Schilit, "Place Lab: Device Positioning Using Radio Beacons in the Wild," in Proc. of the 3rd Int. Conf. on Pervasive Computing (Percom), March 2005.

[4] M. B. Kjaergaard, "A Taxonomy for Radio Location Fingerprinting," in Proc. of the 3rd Int. Symposium on Location- andContext-Awareness (LoCA), Sept. 2007.

[5] T. King, T. Haenselmann, and W. Effelsberg, "Deployment, Calibration, and Measurement Factors for Position Errors in 802.11-based Indoor Positioning Systems," in Proc. of the 3rd Int. Symposium on Locationand Context-Awareness (LoCA), Sept. 2007.

[6] R. Want, A. Hopper, V. Falcăo, and J. Gibbons, "The active badge location system," ACM Transactions on Information Systems, Jan. 1992.

[7] A. Ward, A. Jones, and A. Hopper, "A new location technique for the active office," IEEE Personal Communications, vol. 4, Oct. 1997.

[8] L. M. Ni, Y. Liu, Y. C. Lau, and A. P. Patil, "LANDMARC: indoor location sensing using active RFID," Wireless Networks, Nov. 2004.

[9] V. Bychkovsky, B. Hull, A. Miu, H. Balakrishnan, and S. Madden, "A Measurement Study of Vehicular Internet Access Using In Situ Wi-Fi Networks," in Proc. of the 12th Int. Conf. on Mobile Computing and Networking (MobiCom), Sept. 2006.

[10] T. S. Rappaport, Wireless Communications: Principles and Practice, 2nd ed. Prentice Hall PTR, Dec. 2001.

[11] P. Bahl and V. N. Padmanabhan, "RADAR: An In-Building RF-based User Location and Tracking System," in Proc. of the 19th Int. Conf. on Computer Communications (InfoCom), March 2000.

[12] M. Youssef and A. Agrawala, "On the Optimality of WLAN Location Determination Systems," in Proc. of the Communication Networks and Distributed Systems Modeling and Simulation Conference (CNDS), Jan. 2004. 
[13] A. Haeberlen, E. Flannery, A. M. Ladd, A. Rudys, D. S. Wallach, and L. E. Kavraki, "Practical robust localization over large-scale 802.11 wireless networks," in Proc. of the 10th Int. Conf. on Mobile Computing and Networking (MobiCom), Sept. 2004.

[14] M. Youssef and A. Agrawala, "The Horus WLAN location determination system," in Proc. of the 3rd Int. Conf. on Mobile systems, applications, and services (MobiSys). ACM, 2005.

[15] Widyawan, M. Klepal, and S. Beauregard, "A novel backtracking particle filter for pattern matching indoor localization," in MELT '08: Proc. of the first ACM Int. workshop on Mobile entity localization and tracking in GPS-less environments. ACM, 2008.

[16] A. Varshavsky, A. LaMarca, J. Hightower, and E. de Lara, "The skyloc floor localization system," in Proc. of the 5th Int. Conf. on Pervasive Computing and Communications (Percom), March 2007.

[17] T. King, S. Kopf, T. Haenselmann, C. Lubberger, and W. Effelsberg, "Compass: A probabilistic indoor positioning system based on 802.11 and digital compasses," in Proc. of the First ACM Int. Workshop on Wireless Network Testbeds, Experimental evaluation and CHaracterization (WiNTECH). ACM Press, Sept. 2006.

[18] D. Dearman, A. Varshavsky, E. de Lara, and K. N. Truong, "An exploration of location error estimation," in Proc. of the 9th Int. Conf. on Ubiquitous Computing, 2007.

[19] H. Lemelson, M. B. Kjrgaard, R. Hansen, and T. King, "Error estimation for indoor 802.11 location fingerprinting," in Proc. of the Fourth Int. Symposium on Location and Context Awareness (LoCA 2009), Mai 2009.

[20] T. King and M. B. Kjrgaard, "Composcan: Adaptive scanning for efficient concurrent communications and positioning with 802.11," in Proc. of the 6th Int. Conf. on Mobile Systems, Applications, and Services (MobiSys), June 2008.

[21] H. Lemelson, T. King, and W. Effelsberg, "Pre-processing of fingerprints to improve the positioning accuracy of 802.11-based positioning systems," in Proc. of the 1st Int. Workshop on Mobile Entity Localization and Tracking in GPS-less Environments (MELT), Sept. 2008.

[22] P. Bolliger, "Redpin - adaptive, zero-configuration indoor localization through user collaboration," in MELT '08: Proc. of the first ACM Int. workshop on Mobile entity localization and tracking in GPS-less environments. ACM, 2008.

[23] X. Chai and Q. Yang, "Reducing the calibration effort for location estimation using unlabeled samples," in Proc. of the 3rd Int. Conf. on Pervasive Computing and Communications (PerCom), March 2005.

[24] A. P. Dempster, N. M. Laird, and D. B. Rubin, "Maximum likelihood from incomplete data via the em algorithm," Journal of the Royal Statistical Society. Series B (Methodological), vol. 39, no. 1, 1977.

[25] Y. Ji, S. Biaz, S. Pandey, and P. Agrawal, "ARIADNE: a dynamic indoor signal map construction and localization system," in Proc. of the 4th Int. Conf. on Mobile systems, applications and services (MobiSys), June 2006.

[26] T. King, T. Butter, M. Brantner, S. Kopf, T. Haenselmann, A. Biskop, A. Färber, and W. Effelsberg, "Distribution of Fingerprints for 802.11based Positioning Systems," in Proc. of the 8th Int. Conf. on Mobile Data Management (MDM), Mannheim, Germany, May 2007. 